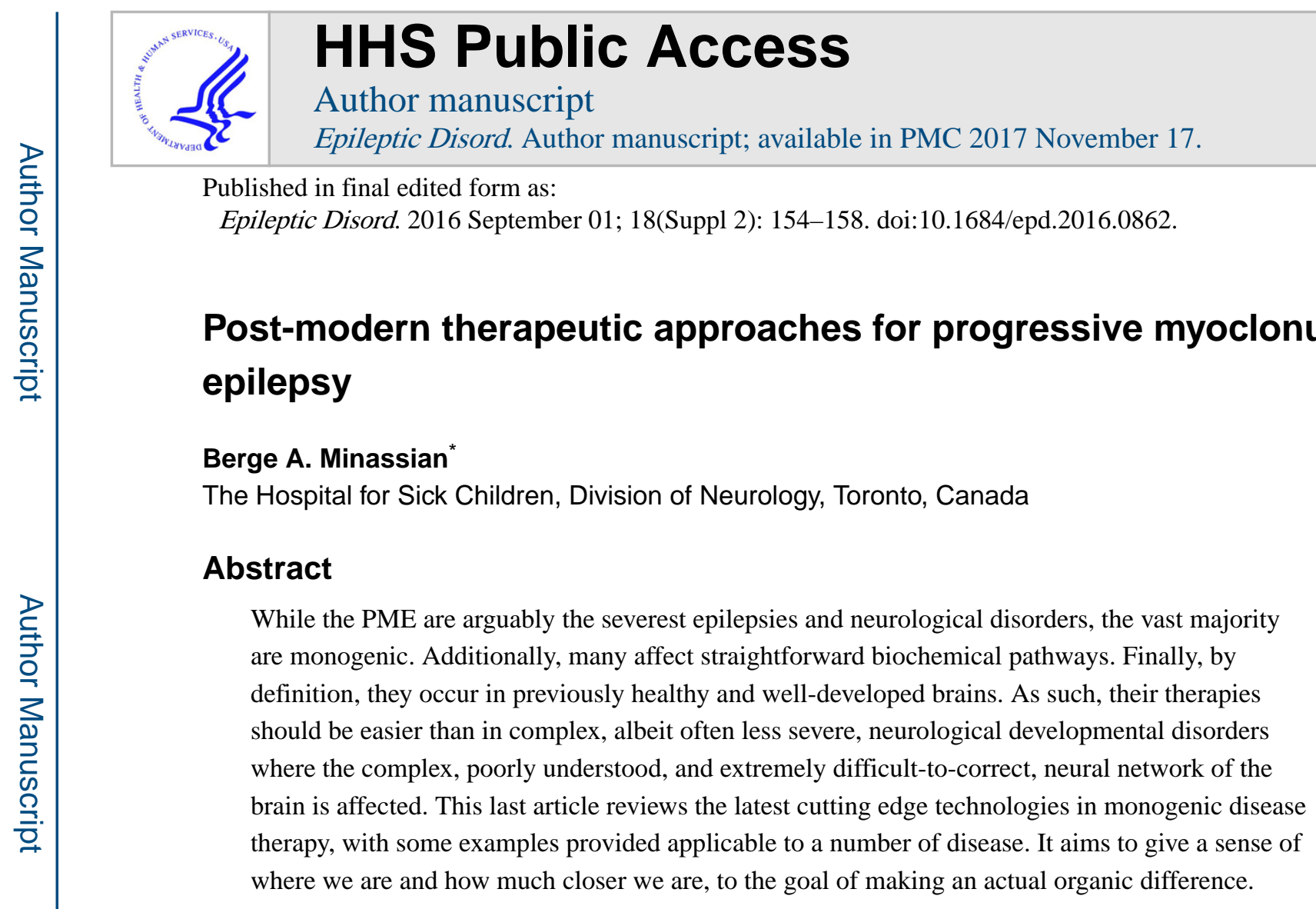

\title{
Keywords
}

Cas9; CRISPR; gene therapy; AAV9; small molecule; progressive myoclonus epilepsies

By and large, progressive myoclonus epilepsies (PMEs) described in this supplement (Minassian et al., 2016) are devastating and can be lethal. However, most are monogenic, rendering them much more amenable to future therapies relative to other complex diseases, for two reasons; firstly, the pathophysiology is easier to understand, and secondly, therapy may be targeted to a single gene, function, or pathway. In this article, some of the cuttingedge therapeutic modalities applicable to monogenic brain diseases is reviewed. In each case, one example is provided, simply as an illustration. It is not possible to provide a comprehensive review since even as this state-of-the-art supplement is being published, discoveries are being made regarding new molecular targeting and delivery systems.

\section{SMALL MOLECULES}

We are accustomed to therapy in the form of a pill ingested daily, the active compound of which travels through the gut and blood, crosses the blood-brain barrier (BBB), and enters cells to impart therapeutic change. The size of small molecules is their abiding therapeutic advantage for PMEs and other brain diseases, and size matters regarding passage through the BBB, but of course, small size is not all it takes. For example, the presence of a monoester

\footnotetext{
*Correspondence: Berge A. Minassian The Hospital for Sick Children, Division of Neurology, 555 University Avenue, Toronto, Ontario, M5G 1X8, Canada.

DISCLOSURES

The author has no conflict of interest to disclose.
} 
(phosphate) bound to a small molecule prevents it from not only crossing the BBB, but even cell membranes.

However, a small molecule will not replace a gene function, but could, as a chaperone, stabilize a gene product which is present but simply misfolded and thus non-functional. In other words, for diseases where at least one of the mutations is a missense mutation that deconvolutes or otherwise destabilizes the tertiary structure of a disease gene product, a small chaperone molecule may stabilize the structure to a sufficient extent to render it functional, effectively above a clinical threshold.

Tay-Sachs disease is a neurodegenerative disease due to mutations in the hexosaminidase A (HEXA) gene. In its classic form, it is an infantile disease that is fatal by age 2 or 3 . However, some "mild" mutations are associated with a milder neurodegenerative course. The FDA-approved antimalarial BBB-penetrant agent, pyrimethamine, was shown to stabilize certain HEXA missense mutants in fibroblasts of patients with late-onset Tay-Sachs disease, and raise enzymatic activity threefold (Maegawa et al., 2007). This led to a pilot clinical study in 4 patients in whom HEXA activity rose 2.24 fold. The rise was unfortunately not sustained and the study was clinically unsuccessful (Osher et al., 2015), but nonetheless this was a start for an otherwise intractable disease.

While small molecules cannot replace gene functions, they can very well affect other parts of a disease pathway, whether metabolic or not, or a second messenger. Often, the absence of a gene causes the activity of a downstream protein to be up or down-regulated, which when corrected through interference by a small molecule, even partially, may revert disease pathogenesis and be therapeutic. mTOR pathway interference with rapamycin and its analogues is a case in point. Tuberous sclerosis and several other epilepsies are caused by genetic disruptions of this pathway, leading to focal or more widespread dysplasias and tumours (usually benign), which drive epilepsy. This therapy, which partially regulates the pathway and presents an entirely novel, more direct mechanism, therefore provides intrinsic support as treatment for these epilepsies (Ricos et al., 2015).

Identifying a small molecule drug commonly requires establishment of in vitro assays; in vitro (biochemical) and/or cell culture assays. These assays are based on the associated molecular or biochemical disease pathway(s) which are investigated through standard gene function-based research. The assays are used to screen ever-expanding libraries of small molecules in order to identify, by chance, one or more that may affect the assay in the desired way. Huge amounts of work follow in translating 'hits' to something useful. This involves establishing safety and efficacy in animal models, mouse and larger animals, BBB passage, and other pharmacological aspects. Usually, the initial 'hit' is less than optimal, and a prolonged process of medicinal chemistry towards optimization follows, until eventually a drug can be ready for testing in human subjects. This type of work is long and expensive. However, the infrastructure is increasingly in place in many universities, and even more so in pharmaceutical companies. The latter appear to be moving very much in the direction of developing drugs for orphan diseases. These are usually so devastating that families and societies will incur the costs of these drugs which will always be priced high, in part, to recover the large investment of development. 
While "chance" through high-throughput screening is the common and strongest approach to identify interfering molecules, the vast expansion of crystal structures of proteins available as well as the bioinformatics tools to study these structures and their functions, are allowing intelligent drug design, e.g. in silico development of chemicals that might fit an enzyme's active site. This is presently in its infancy, but clearly holds much promise. One of the biggest problems with small molecules is their usual incomplete selectivity, i.e. their interference with other targets, leading to side effects. This too could partially be managed in the future with complete understanding of protein structures and even stronger informatics tools. Clearly, oral drugs are the most desired therapeutic approach due to non-invasiveness, but drugs are neither permanent solutions, nor, for the moment, easy to tailor to ensure a high degree of target specificity. As such, other approaches are needed, as discussed below. Some are invasive, but this is mitigated by the huge need for them, given the devastating nature of the PMEs.

\section{TARGETING RNA}

As well as targeting proteins, it is possible to target messenger RNA (mRNA). The same general principles hold, in that it is generally much easier to downregulate mRNA than increase it, and the same issues regarding BBB passage exist. There are a number of antiRNA approaches, the most advanced of which is the use of antisense oligonucleotides (ASOs). These oligonucleotides are designed to interfere with an mRNA and downregulate it. One of these is already on the market as a drug for familial hyperlipidaemia (Phillips et al., 2015).

There are currently many ASOs in clinical trials for various neurological diseases (e.g. Huntington disease and spinocerebellar ataxia [Keiser et al., 2015]), but all share the same problem in that they do not cross the BBB and require intra-cerebrospinal fluid administration. This problem is mitigated by specific chemical properties that allow their longevity, and the patient does not require an injection more than once every two months. This is clearly still far from ideal, but for the moment, is the best available treatment. Interestingly, it has been reported that ASOs can actually activate a function which has been lost.

The most common cause of spinal muscular atrophy (SMA) is loss-of-function mutation of the $S M N 1$ gene. It so happens that evolution has led to the $S M N 1$ gene being located adjacent to the $S M N 2$ gene. In SMA, SMN2 is also generally inactive due to a sequence that leads to skipping over exon 7. ASOs have been designed to interfere with this skipping of exon 7 splicing, and thus lead to inclusion of this exon, thus rendering SMN2 functional. This "drug" is presently in an advanced clinical trial (Faravelli et al., 2015).

\section{TARGETING DNA}

Bacteria, of course have an immune system; how else would they survive the onslaught of viruses over millions of years? This immunity consists of generating guide RNAs that specifically target enzymes (e.g. Cas9) to viral genomes. The Cas9 then nicks and inactivates the viral DNA. 
Impressive recent studies have addressed the following questions. Could Cas9 cut human DNA? The answer is yes. Could Cas9 be directed by specific guide RNA sequences to specific sites in the human genome to cut specific genes? Yes. Would the human genome be able to mend itself after being cut? Yes. Does this correction maintain the coding frame of a target gene? No. In toto, this CRISPR (which refers to the guide RNA)/Cas9 system can specifically target a desired gene and break it, causing the cell's DNA repair system to mend the chromosomal break, however, usually not in-frame, i.e. the targeted gene is knocked out, but the chromosome is otherwise untouched (Ran et al., 2015).

The major advantage of this genome editing approach is that it is permanent. Presently, the system only knocks out genes. If a disease pathway is identified in which a protein function in the pathway is increased, and its elimination would correct pathogenesis, then CRISPR/ Cas9 could be utilized to remove this protein and treat the disease. The greatest therapeutic promise of the system, however, is in future possibilities. If the system can be targeted to a mutant gene, specifically to the mutation site, such that the mutation is removed and replaced with normal sequence, then the disease would be corrected at the source.

There are countless difficulties to overcome before the CRISPR system delivers. Firstly, Cas9 and guide RNA have to be delivered to the brain, no small feat as this is a large protein. It would be possible to deliver CRISPR/Cas9 through viral gene therapy, but the gene for the commonly used Cas9, from Strep. viridans, is too large to fit in the AAV viral vectors that have the greatest promise for gene therapy. This problem, though, appears to have been solved with the identification of a smaller, but equally effective, Cas9 gene from Staph. aureus, which does fit in AAV particles (Ran et al., 2015).

Another problem is the need to deliver to large numbers of neurons, i.e. 'edit' most neurons, which is a limitation, though perhaps not serious, of present viral vectors (see next section). Then there is the likely immunogenicity of Cas9 delivered to humans. However, Cas9 will not need to be delivered many times, because its effect would be permanent. As such, the patient could be immunosuppressed during the treatment.

Another problem is the risks associated with off-target nicking. While bioinformatics is used to design specific CRISPRs against specific sequences, the genome is vast enough that there is at least the risk of off-target cutting, with potential for 'side-effects', including oncogenesis.

Off-target effects, however, from most recent data, appear to be not nearly as concerning as initially thought (Iyer et al., 2015). In addition, there is, of course, the looming issue that gene deletion as a therapeutic approach would be useful in only very few situations. What is desired is mutation correction, and the CRISPR system is not quite there yet. There have been successes, but correction of gene mutation remains inefficient and much work is required to optimize it. However, if this system proves to be successful, it would allow, as mentioned, correction of the root of the problem in essentially all the diseases described in this book. An important step forward has been taken recently in this endeavour. Yet another bacterial DNA nickase has been identified, which, unlike Cas9, cleaves DNA via a staggered 
cut, leading to overhanging ends, which should make the replacement of a deleted segment by a corrected sequence much easier (Zetsche et al., 2015).

\section{GENE THERAPY}

Most of the conditions described in this supplement are autosomal recessive diseases caused by a loss of function of single genes. As such, they can potentially be remedied by replacing the gene function. Gene replacement therapy stalled for a long time in the 1990s due to premature use of immunogenic viruses for gene delivery with subsequent harm to patients. However, gradual careful work has brought to the fore the adeno-associated viruses, in particular AAV9 and AAVrh10, which have superb properties. These viruses are normally present in the human brain, implying natural BBB passage capability and low immunogenicity. However, low immunogenicity does not mean no immunogenicity. The high load of virus that needs to be given generates immune reactions in animal models, although, presumably, this problem can be averted by immunosuppression during treatment. AAV viruses can package genes of average size, which means they are useful, potentially, for most diseases. The DNA vectors are not silenced, leading to persistent expression. Their DNA does not integrate into the human genome, i.e. there is low to no risk of interfering with genomic DNA, including tumour suppressor genes. Finally, AAV9 is capable of delivering its cargo to a large proportion of cells, in particular, when injected in the CSF (Meyer et al., 2015).

Gene therapy is indeed in resurgence and it is hoped the technical difficulties will continue to be overcome, to allow gene replacement, and, as discussed above, gene correction. In fact, clinical trials are ongoing for multiple diseases, including some neuronal ceroid lipofuscinoses reviewed in this supplement, as well as SMA, as mentioned above. While the ASO approach for SMA aims at activating SMN2, the AAV-delivered gene therapy approach aims to replace under-expressed $S M N 1$. Over a dozen babies with this fatal disease have been injected so far with virus carrying $S M N 1$, and they are doing well (B. Kaspar, personal communication); publication of this major potential therapeutic success by the group which has been working on it for many years is anticipated (Mandel, Kaspar and colleagues).

While gene therapy mostly utilizes viruses, it must be noted that there are other approaches. These include packaging the gene of interest in liposomes, which merge with membranes and can potentially make their way through the BBB to neurons. Immunoliposomes are another development on this concept. Here, the liposome membrane contains an antibody that allows it to bind to membrane receptors that are then internalized, thus supporting transduction into the brain.

\section{PROTEIN REPLACEMENT THERAPY}

In this last section, we touch on the possibility of directly replacing the missing protein, instead of its gene. Multiple approaches are used. This relies, as for immunoliposomes, on antibodies against the transferrin receptor or other proteins. The antibody is covalently bound to the protein to be delivered, and once it interacts with its target, it and the protein 
are internalized into vesicles, which then merge with lysosomes. As such, this type of therapy would be useful for lysosomal diseases, which make up a large part of PME.

Another exciting possibility is the use of diphtheria toxin. This toxin binds a receptor on the endothelial cell membrane and is internalized into a vesicle into the endothelial cells that make up the BBB. This vesicle translocates to the other side of the cells and releases the toxin into the CSF space. The toxin then binds to its receptor at the surface of neurons, is again internalized into a vesicle, but this time it is injected out of the vesicle into the neuronal cytoplasm, where it blocks protein synthesis and kills the neuron. It was shown that the toxicity of this toxin can be completely eliminated by a point mutation, whilst retaining its translocation properties. Finally, it was shown that a cargo protein, almost of any size, can be attached to this toxin and co-delivered to cells (so far in cell culture experiments only) with maintained function of the delivered protein (Auger et al., 2015).

There are other post-genetic era therapeutic approaches in the works, and indeed a lot of excitement that the expression of certain genes will follow Mendelian genetics. For example, for Lafora disease, one of the prototypical PMEs described in an earlier chapter of this supplement (Turnbull et al., 2016), progress in understanding the disease has allowed us to 'cure' Lafora mice simply by reducing the amount of glycogen synthase (GS), the enzyme that gives rise to the neurotoxic Lafora bodies that underlie the disease. As such, we are presently applying almost all the methods above to attempt to find a therapy for this horrendous condition. This involves screening for small molecule inhibitors of GS, using ASOs against GS and its activator protein PTG, experimenting with CRISPRs against the latter two proteins, using AAV9 to replace the two respective disease-causing genes, and using inactivated diphtheria toxin-mediated delivery of amylase to mouse brain. Amylase is the only enzyme known to digest Lafora bodies. We have already shown that we are able to deliver amylase to cells in culture in this fashion and that amylase remains functional after crossing the cell membrane (Auger et al., 2015). This disease, which we are particularly familiar with, exemplifies the immense possibilities ahead for all our patients with any form of PME.

\section{References}

[Auger et al 2015]. Auger A, Park M, Nitschke F. Efficient delivery of structurally diverse protein cargo into mammalian cells by a bacterial toxin. Mol Pharm. 2015; 12:2962-29671. [PubMed: 26103531]

[Faravelli et al 2015]. Faravelli I, Nizzardo M, Comi GP, Corti S. Spinal muscular atrophy-recent therapeutic advances for an old challenge. Nat Rev Neurol. 2015; 11:351-359. [PubMed: 25986506]

[Iyer et al 2015]. Iyer V, Shen B, Zhang W. Off-target mutations are rare in Cas9-modified mice. Nat Methods. 2015; 12:479. [PubMed: 26020497]

[Keiser et al 2015]. Keiser MS, Kordasiewicz HB, McBride JL. Gene suppression strategies for dominantly inherited neurodegenerative diseases: lessons from Huntington's disease and spinocerebellar ataxia. Hum Mol Genet. 2015; 25:R53-64. [PubMed: 26503961]

[Maegawa et al 2007]. Maegawa GH, Tropak M, Buttner J. Pyrimethamine as a potential pharmacological chaperone for late-onset forms of GM2 gangliosidosis. J Biol Chem. 2007; 282:9150-9161. [PubMed: 17237499]

Epileptic Disord. Author manuscript; available in PMC 2017 November 17. 
[Meyer et al 2015]. Meyer K, Ferraiuolo L, Schmelzer L. Improving single injection CSF delivery of AAV9-mediated gene therapy for SMA: a dose-response study in mice and non-human primates. Mol Ther. 2015; 23:477-487. [PubMed: 25358252]

[Minassian et al 2016]. Minassian BA, Striano P, Avanzini G. Progressive Myoclonus Epilepsies: State-of-the-Art. Epileptic Disord. 2016; 18(Suppl 2):S1-S158.

[Osher et al 2015]. Osher E, Fattal-Valevski A, Sagie L. Effect of cyclic, low dose pyrimethamine treatment in patients with late onset Tay Sachs: an open label extended pilot study. Orphanet J Rare Dis. 2015; 10:45. [PubMed: 25896637]

[Phillips et al 2015]. Phillips MI, Costales J, Lee RJ, Oliveira E, Burns AB. Antisense therapy for cardiovascular diseases. Curr Pharm Des. 2015; 21:4417-4426. [PubMed: 26234793]

[Ran et al 2015]. Ran FA, Cong L, Yan WX. genome editing using Cas9. Nature. 2015; 520:186-191. In vivoStaphylococcus aureus. [PubMed: 25830891]

[Ricos et al 2015]. Ricos MG, Hodgson BL, Pippucci T. Mutations in the mTOR pathway regulators NPRL2 and NPRL3 cause focal epilepsy. Ann Neurol. 2015; 79:120-131. [PubMed: 26505888]

[Turnbull et al 2016]. Turnbull J, Striano P, Genton P, Carpenter S, Ackerley CA, Minassian BA. Lafora Disease. Epileptic Disord. 2016; 18(Suppl 2):S38-62.

[Zetsche et al 2015]. Zetsche B, Gootenberg JS, Abudayyeh OO. Cpf1 is a single RNA-guided endonuclease of a class 2 CRISPR-Cas system. Cell. 2015; 163:759-771. [PubMed: 26422227] 